INPLASY

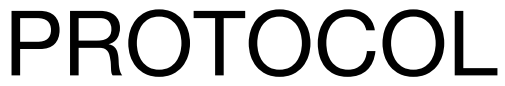

To cite: Wali et al. Clinical and Histological impacts of radiotherapy on the dental pulp: A systematic review. Inplasy protocol 202130030. doi:

10.37766/inplasy2021.3.0030

Received: 09 March 2021

Published: 09 March 2021

Corresponding author:

Rana Wali

rana.wali@hotmail.com

Author Affiliation:

Guy's Hospital

Support: Not applicable.

Review Stage at time of this submission: The review has not yet started.

Conflicts of interest:

None declared.

\section{Clinical and Histological impacts of radiotherapy on the dental pulp: $A$ systematic review}

\author{
Wali, R'; Sacco, R²; Patel, V3.
}

Review question / Objective: Question: What are the clinical and histopathological effects of radiotherapy on the dental pulp of non-carious teeth in head and neck cancer patients? P: Any (no limits of age) patients previously or currently undergoing radiotherapy for head and neck cancer; I: not applicable; C: not applicable; O: pulpal response to sensibility/ vitality testing and histopathological findings; S: Randomized controlled trials, Case-controlled trials, Cohort studies (prospective and or retrospective), Case series, Case Report. Condition being studied: It has been suggested that acute hematologic abnormalities and thrombosis in the microcirculation start occurring at $1 \mathrm{~Gy}$ radiation. Furthermore, ionizing radiation causes chemical injuries in the tissues, and therefore radiation therapy may be the reason for changes in microcirculation. In the dental pulp, this may manifest clinically as reduced or absent response to sensibility testing, or histopathologically as microscopic alteration of the dental pulp. This systematic review aims to investigate if teeth located in the irradiation field are subject to a reduction in circulation through pulpal tissue. We intend to assess the current evidence surrounding the effect of radiation induced hypo-vascularity on the clinical and/or histopathological status of the dentition.

INPLASY registration number: This protocol was registered with the International Platform of Registered Systematic Review and Meta-Analysis Protocols (INPLASY) on 09 March 2021 and was last updated on 09 March 2021 (registration number INPLASY202130030).

\section{INTRODUCTION}

Review question / Objective: Question: What are the clinical and histopathological effects of radiotherapy on the dental pulp of non-carious teeth in head and neck cancer patients? P: Any (no limits of age) patients previously or currently undergoing radiotherapy for head and neck cancer; I: not applicable; C: not applicable; O: pulpal 
response to sensibility/ vitality testing and histopathological findings; S: Randomized controlled trials, Case-controlled trials, Cohort studies (prospective and or retrospective), Case series, Case Report.

Rationale: Radiotherapy (RT) is known to negatively influence the structural morphology of tissues. Patients who have undergone RT for a range of malignancies such as breast cancer, lymphoma and head and neck cancer (H\&NC) are at risk of developing macro and microvascular changes which can lead to significant clinical consequences. Despite dramatic advances in the field of radiation oncology and the development of Intensitymodulated radiotherapy (IMRT), radiation injury to the blood vessels remains a modern-day clinical problem. Blood vessels have a crucial role in the early and late effects of RT as they are the most affected component, and serve as a gateway to a vast array of biochemical mechanisms that influence further soft and hard tissue modifications. The pulpal status of a patient's dentition post-RT is key to their long term treatment planning and management. Knowledge of the effects of RT on pulpal status may enhance the predictability of pre-RT dental assessment and treatment planning. Demonstration of a dose and/or time-dependent relationship between pulpal status and clinical and/or histopathological findings could help build a basis for long-term prognostic factors for the dentition of H\&NC patients. Conclusive data regarding the effects of $\mathrm{RT}$ on the microvasculature of the dental pulp is lacking. The current systematic review aims to investigate the available evidence regarding dose and time-effect relationship involved in radiation-induced microvascular changes within the dental pulp, and the clinical and histopathological manifestations of this. Hypothesis: RT effects the microvasculature and components of the dental pulp, subsequently reducing clinical response to sensibility/vitality testing and inducing histopathological changes in the pulp. We also hypothesise that the effects of radiotherapy on the dental pulp are time and dose dependent.
Condition being studied: It has been suggested that acute hematologic abnormalities and thrombosis in the microcirculation start occurring at $1 \mathrm{~Gy}$ radiation. Furthermore, ionizing radiation causes chemical injuries in the tissues, and therefore radiation therapy may be the reason for changes in microcirculation. In the dental pulp, this may manifest clinically as reduced or absent response to sensibility testing, or histopathologically as microscopic alteration of the dental pulp. This systematic review aims to investigate if teeth located in the irradiation field are subject to a reduction in circulation through pulpal tissue. We intend to assess the current evidence surrounding the effect of radiation induced hypo-vascularity on the clinical and/or histopathological status of the dentition.

\section{METHODS}

Search strategy: A multi-database search (PubMed, MEDLINE, EMBASE and CINAHL) will be performed to identify related multilanguage publications. Data will be extracted from relevant papers and analysed according the outcomes selected in this review. A search strategy for all databases will be used as follows: 1 . Radiation [MeSH Terms] OR Radiotherapy [MeSH Terms] OR Radiation treatment [MeSH Terms] OR RT [MeSH Terms] OR IMRT [MeSH Terms] OR Three-dimensional conformal radiation therapy [MeSH Terms] OR 3DCRT [MeSH Terms] OR Volumetric modulated arc therapy [MeSH Terms] OR VMAT [MeSH Terms] OR stereotactic radiotherapy [MeSH Terms] OR SRT [MeSH Terms] OR Brachytherapy [MeSH Terms] OR Intraoperative radiation therapy [MeSH Terms] OR IORT [MeSH Terms]. 2. Head and neck [MeSH Terms] OR Head and neck cancer [MeSH Terms] OR Head and neck oncology [MeSH Terms] OR Oral cancer [MeSH Terms] Pharyngeal cancer [MeSH Terms]. 3. Pulp [MeSH Terms] OR Dental pulp [MeSH Terms] OR Tooth pulp [MeSH Terms] OR Root canal pulp [MeSH Terms] OR Teeth [MeSH Terms]. 4. 1 and 2 and 3. The search strategy includes appropriate changes in the keywords and follows the syntax rules of each database. All articles 
published from inception of each library, to the date of search (predicted March 2021) will be screened and duplicates will be removed. A three-stage screening approach will be used to ensure precision, and safeguard the quality of the search. The screening of titles and abstracts will be carried out independently by two authors (RW, VP) to eliminate any irrelevant material (i.e., reviews, animal studies, non-clinical studies). Disagreements will be resolved by discussion with a third author (RS) until a consensus is reached. An additional manual search will be performed to ensure that all relevant and available research papers are included in the review.

Participant or population: Our population of interest is H\&NC patients of any age or gender, treated with radiotherapy with curative intent. Teeth included will be those tested clinically and/or histologically for the effects of radiotherapy on the dental pulp. Teeth affected by caries, apical or pulpal pathology, significant periodontal bone loss or a history of trauma will be excluded to limit the potential for confounding factors. Teeth that have been investigated for nonpulpal effects only (e.g. enamel/ dentine/ cementum) will be excluded. All H\&NC sites and stages will be included and there will be no restrictions on the form or modality of radiotherapy involved. There will be no restrictions on population demographics.

Intervention: The current question will be addressed by non-interventional studies.

Comparator: The current question will be addressed by non-interventional studies.

Study designs to be included: All human subject published or unpublished randomized control trials, case-control trials, cohort studies (prospective and/or retrospective), case series, and case reports.

Eligibility criteria: Inclusion: - No language restrictions - Studies involving human subjects with non-cairous teeth post radiotherapy - All forms of radiotherapy and delivery modalities - Studies in which the pulpal investigations have been carried out in vivo - All forms of well-defined sensibility testing e.g. (ethyl chloride/ EndoFrost/ pulse oximetry) - Studies in which the pulpal investigations have been carried out in vitro - Studies with any welldefined histological/microscopic markers of vascularity - All study designs with a clearly defined method. Exclusion: Studies involving animal subjects Radiotherapy given for non-head and neck cancers - Studies involving non-ionising radiation e.g. laser - Studies in which the involved teeth are deciduous - Carious teeth or teeth with apical/ pulpal pathology, significant $(>50 \%)$ periodontal bone loss or a history of trauma - Studies that do not investigate the pulpal effects of radiotherapy

Information sources: The intended information sources for the current systematic review are: - Electronic databases (PubMed, MEDLINE, EMBASE, CINAHL) - Contact with authors where further information is required.

Main outcome(s): The main outcomes of the review are: - The effects of radiotherapy on pulpal response to sensibility/ vitality testing of teeth in an irradiated field. - The histopathological effects of radiotherapy on the microvasculature and components of the dental pulp.

Additional outcome(s): - Time and/or dosedependent effects of radiotherapy on the dental pulp (clinical and histological); Predisposing factors to clinical/ histopathological pulpal changes (smoking, pre-extsiting periodontitis etc); - Gender preference (is there any predilection amongst genders to clinical/ histopathological changes); - Teeth more likely to have clinical and/or histopathological changes (molars, premolars, incisors etc).

Data management: Data extracted from the studies will be completed using a predefined and standardized Microsoft Excel form. This will be done by two investigators (RW, VP) independently. Any discrepancies will be resolved using a third investigator (RS) and conflicts resolved by 
discussion. A data extraction table will be constructed to include study results and conclusions. This will be developed by taking into account the homogeneity of the studies and their degree of comparability. A database for quality assessment of the studies will be constructed for presentation and comparative purposes.

Quality assessment / Risk of bias analysis: Two review authors will appraise the risk of bias in the included study with the Cochrane Handbook for Systematic Reviews of Interventions (RoB2) in case of any randomised controlled trials found. For case-control studies they will use the Risk of Bias in Non-randomised Studies - of Interventions (ROBINS-I). The authors will use the consensus-based clinical case reporting guidelines development (CARE checklist) and the Strengthening the Reporting of Observational Studies in Epidemiology (STROBE) checklist for case series and longitudinal studies.

Strategy of data synthesis: This systematic review will not adopt any restrictions on minimum number of studies or heterogeneity of the studies. In case of heterogeneity of the cohort of patients and studies, descriptive statistics will be used to analyse and present the data.

Subgroup analysis: We anticipate the data are little and heterogenic; statistical analysis will most likely be descriptive.

Sensitivity analysis: Many issues suitable for sensitivity analysis may only be identified during the review process. Where sensitivity analyses identifies missing information that may influence the findings of the review, an attempt will be made to contact trial authors. If this cannot be achieved, results will be interpreted with a degree of caution. If sufficient data are extracted, a sensitivity analysis will be conducted to check the stability of the outcome results by excluding low methodological quality or high risk of bias studies.
Language: There will be no language restrictions on studies included in the systematic review.

Country(ies) involved: United Kingdom.

Keywords: Pulp; radiotherapy; head and neck cancer; histology; histopathology.

Dissemination plans: We intend to disseminate the findings of our systematic review through publication in a relevant journal, and presentation at relevant conferences.

Contributions of each author:

Author 1 - Rana Wali - The authour is the primary investigator and will draft the manuscript.

Email: rana.wali@gstt.nhs.uk

Author 2 - Roberto Sacco - The author has provided expertise in systematic reviews and will be approached for discrepencies in article selection for inclusion in the systematic review. The author contributed to development of a search strategy and the risk of bias assessment strategy.

Email: roberto.sacco@manchester.ac.uk Author 3 - Vinod Patel - The author will be another independent investigator and will provide expertise on the field of the dental effects of radiotherapy.

Email: vinod.patel@gstt.nhs.uk 